\title{
Magnetic and transport signatures of Rashba spin-orbit coupling on the ferromagnetic Kondo lattice model in two dimensions
}

\author{
Giovany A. Meza and José A. Riera \\ Instituto de Física Rosario y Departamento de Física, Universidad Nacional de Rosario, Rosario, Argentina
}

(Received 5 May 2014; revised manuscript received 21 July 2014; published 11 August 2014)

\begin{abstract}
Motivated by emergent phenomena at oxide surfaces and interfaces, particularly those involving transition metal oxides with perovskite crystal structure such as $\mathrm{LaTiO}_{3} / \mathrm{SrTiO}_{3}$, we examine the ferromagnetic Kondo lattice model (FKLM) in the presence of a Rashba spin-orbit coupling (RSOC). Using numerical techniques, under the assumption that the electrons on localized orbitals may be treated as classical continuum spins, we compute various charge, spin, and transport properties on square clusters at zero temperature. We find that the main effect of the RSOC is the destruction of the ferromagnetic state present in the FKLM at low electron fillings, with the consequent suppression of conductivity. In addition, near half filling the RSOC leads to a departure of the antiferromagnetic state of the FKLM with a consequent reduction to the intrinsic tendency to electronic phase separation. The interplay between phase separation on one side, and magnetic and transport properties on the other, is carefully analyzed as a function of the RSOC/hopping ratio.
\end{abstract}

DOI: 10.1103/PhysRevB.90.085107

PACS number(s): 71.27.+a, 71.70.Ej, 73.20.-r

\section{INTRODUCTION}

Transition metal oxides (TMOs) are typical strongly correlated systems because of the available $d$ orbitals, and are characterized by a complex interrelation between charge, spin, and orbital degrees of freedom, leading in turn to remarkable properties such as high- $T_{c}$ superconductivity in cuprates and colossal magnetoresistance in manganites. The intensive study of these strongly correlated systems has identified the ultimate origin of those phenomena as caused by the collective behavior of electrons, which could only be captured by appropriate many-body approaches.

Additional emergent phenomena at the interface between strongly correlated materials, and particularly TMOs, or at the surface of such materials, have been revealed even more recently by a number of theoretical and experimental studies [1]. In essence, this exciting new physical phenomena is induced by the breaking of the inversion symmetry, $\mathbf{r} \rightarrow-\mathbf{r}$, at the interface (or surface) itself. Also, it is important to notice that reducing the spatial dimensionality, to two dimensions in the present case, usually enhances the effects of electron correlations as it is well-known in fact by studies on cuprates and other layered perovskites. As a result of the broken inversion symmetry, and due to relativistic considerations, the so-called Rashba effect appears [2], which describes various momentum-dependent spin-splitting processes, including spin currents and the spin-Hall effect $[3,4]$. The relativistic or Rashba spin-orbit coupling (RSOC) is usually stated by the Hamiltonian [5]:

$$
H_{\mathrm{SOC}}=\alpha_{R}(\mathbf{k} \times \sigma) \cdot \hat{z},
$$

where $\mathbf{k}$ is the electron momentum, $\sigma$ the electron spin, and $\hat{z}$ is the unit vector normal to the surface or interface. As emphasized in a rather extensive literature, the Rashba SOC opens new avenues for applications in spintronic devices [6-8]. The Rashba coefficient $\alpha_{R}$ is in principle proportional to the electric field appearing due to the broken inversion symmetry, and in an appropriate device, this electric field can also be tuned by an external gate voltage.
Various types of interfaces between TMOs where RSOC is present have been studied, mostly involving $\mathrm{SrTiO}_{3}$ [9-12], particularly $\mathrm{LaAlO}_{3} / \mathrm{SrTiO}_{3}$ [13-15], but also $\mathrm{LaMnO}_{3} / \mathrm{SrMnO}_{3}$ interfaces [1] have been considered. The contribution of gate tunable RSOC in addition to the in-plane RSOC of broken symmetry origin, has been measured in $\mathrm{LaAlO}_{3} / \mathrm{SrTiO}_{3}$ interfaces [12,14]. A Rashba SOC has also been reported in devices involving magnetic layers such as a Co layer with asymmetric Pt and $\mathrm{AlO}_{x}$ interfaces [16-18]. Experimental indications of Rashba SOC have also been reported at surfaces in $\mathrm{SrTiO}_{3}$ [19] and in $\mathrm{KTaO}_{3}$ [20].

The microscopic description of several transition metal oxides and heavy fermions is achieved through generalizations of the ferromagnetic Kondo lattice model [21-24] (FKLM) also called the double exchange model particularly when the Hund exchange coupling is much larger than the hopping integral. Renewed features and materials to which this model can be applied are discussed in Ref. [26]. For a large Hund coupling, it is known that a metallic ferromagnetic (FM) phase up to a filling $v \sim 0.8$, followed by a tendency towards an antiferromagnetic (AFM) state with semiconducting or insulating properties [25,27-33]. Another issue that has been thoroughly discussed in the context of manganites is the presence of an instability towards phase separation [29,34]. Most of these previous studies have been accomplished using the highly simplifying hypothesis that the electrons in the localized orbitals behave as classical continuum spins, which is a reasonable assumption at least for manganites [35]. This assumption allows the use of a finite-temperature Monte Carlo technique to sample the classical spins [27].

The main goal of the present study is to determine how the Rashba spin-orbit coupling affects the magnetic and transport properties in the two-dimensional (2D) FKLM, particularly in its simplest form when only one conduction electron orbital is included. Although a quantitative study of interfaces in TMOs and hence a comparison with, for example, transport experiments [9] would require a multiorbital model [36], we believe that a first effort to qualitatively understand the effects of the RSOC should start from the single-orbital FKLM, following the program that has been pursued, for example, 
in the study of manganites. In fact, as we will show below, the resulting model Rashba FKLM presents a highly complex interplay between magnetic and transport properties, which deserves a careful study before taking into account more involved multiorbital models. Due to the increased complexity implied by the inclusion of the RSOC, we also concentrate our study to zero temperature, although actually most of the previous studies have dealt with essentially zero-temperature properties. This allows us to study the RSOC in the full range from zero to infinity, with respect to the band hopping parameter. The issue of phase separation will also be addressed in the present effort since experimental indications for this instability on interfaces with strong Rashba SOC have also been reported [37].

The Kondo lattice model supplemented by a Rashba spin-orbit coupling for the conduction electrons on the hexagonal lattice has been recently considered to study topological and Kondo insulating phases [38]. We would also like to stress that the relativistic Rashba SOC is not the on-site or atomic spin-orbit interaction usually discussed in the context of $\mathrm{TbMnO}_{3}$ [39], $\mathrm{Sr}_{2} \mathrm{IrO}_{4}$ [40] or in the perovskitelike Ruddlesden-Popper series of the ruthenates $\mathrm{Sr}_{n+1} \mathrm{Ru}_{n} \mathrm{O}_{3 n+1}$ [41]. This is also the case of recently studied models for the $\mathrm{LaAlO}_{3} / \mathrm{SrTiO}_{3}$ interface [11]. In the model considered in the present work, the RSOC contributes to the kinetic energy and competes with the spin-conserving hopping term [42]. We would like to emphasize that the above mentioned systems where the RSOC takes place in ferromagnetic layers[16-18] can also be modelled by the Rashba FKLM with the localized classical spins playing the role of FM moments. Finally, materials with strong RSOC also display many other interesting features such as the above mentioned topological insulators [43-45], and relativistic Dirac electrons in graphene [46] and other compounds [47,48].

The paper is organized as follows. In Sec. II, we introduce our Rashba ferromagnetic Kondo lattice model. The zerotemperature perturbative MC method is described in Sec. III. Results for magnetic and transport properties, and phase separation, for all electron fillings are presented in Sec. IV A, together with a study of the effect of magnetic fields and spectral functions, which are performed only at quarter filling.

\section{MODEL}

It is well known that in TMOs with perovskite structure, the originally fivefold degenerate $3 d$ orbitals are split into threefold degenerate $t_{2 g}$ orbitals $x y, y z, z x$, and twofold degenerate $e_{g}$ orbitals $x^{2}-y^{2}, 3 z^{2}-r^{2}$. The prototypical TMO compounds where the FKLM was applied are the manganites with occupied $t_{2 g}$ orbitals that appear as localized spins. However in the surface/interfaces of this material the RSOC is somewhat weak, although it has been suggested that the spin-spiral state of orthorhombic manganites is strongly deformed by their relativistic spin-orbit interaction [49]. In $\mathrm{SrTiO}_{3}$, only the $t_{2 g}$ orbitals are partially occupied and involved in both hopping and Rashba processes. However, at the surface/interface, the filling of orbitals could change due to orbital mixing [50,51]. More importantly, itinerant electrons in the $t_{2 g}$ bands of Ti interact with local magnetic moments originating from electrons localized at the interface [13,52], which could be described by a Rashba FKLM as the one here studied. Besides, across an interface, located in the $x y$ plane, due to symmetry constraints, the solely surviving hoppings are those involving $z x / z x, y z / y z$, and $3 z^{2}-r^{2} / 3 z^{2}-r^{2}$ orbitals [1]. There is additional complexity at the interface such as dynamical transfer of electrons from the bulk and location of the interface layer [14,53]. A possible effective Rashba coupling has been proposed for $\mathrm{Sr}_{2} \mathrm{RuO}_{4}$, where a FKLM for its $d^{4}$ orbitals would be appropriate, only within its chiral superconducting state [54]. Finally, the model studied in the present work could be applied to describe the observed tilting of magnetic order in the already mentioned systems containing a Co layer with asymmetric interfaces [16-18]. In this case, the interaction between localized spins $J$ (see below) should be strongly ferromagnetic.

Hence, we believe that important insights on the physics of perovskite interfaces can be obtained from the Rashba FKLM for a single delocalized orbital coupled to classical localized spins defined by the Hamiltonian:

$$
\begin{aligned}
H_{1 o}= & H_{0}+H_{\mathrm{int}} \\
H_{0}= & -t_{0} \sum_{\langle l, m\rangle, \sigma}\left(c_{l \sigma}^{\dagger} c_{m \sigma}+H . c .\right)+\lambda_{S O} \sum_{l}\left[c_{l+x \downarrow}^{\dagger} c_{l \uparrow}\right. \\
& \left.-c_{l+x \uparrow}^{\dagger} c_{l \downarrow}+i\left(c_{l+y \downarrow}^{\dagger} c_{l \uparrow}+c_{l+y \uparrow}^{\dagger} c_{l \downarrow}\right)+\text { H.c. }\right] \\
H_{\mathrm{int}}= & -J_{H} \sum_{l} \mathbf{S}_{l} \cdot \mathbf{s}_{l}+U \sum_{l} n_{l \uparrow} n_{l \downarrow}+J \sum_{\langle l, m\rangle} \mathbf{S}_{l} \cdot \mathbf{S}_{m} .
\end{aligned}
$$

The first term in the noninteracting part $H_{0}$, is the usual hopping term, $H_{0, \text { hop }}$, and the second one corresponds to the RSOC, $H_{0, S O}$, assuming a square lattice in the $x y$ plane ( $z$ is the spin quantization axis) [55]. The first term in the interacting part of the Hamiltonian $H_{\text {int }}$ is the ferromagnetic Hund term, $H_{H}$, between localized $\mathbf{S}_{l}$ and conduction electron $\mathbf{s}_{l}$ spins. The second term is the Hubbard repulsion between conduction electrons, $H_{U}$, and the last one corresponds to the antiferromagnetic exchange Hamiltonian between localized spins, $H_{J}$. This last term is due to virtual processes involving various hoppings and the Coulomb interaction $U$, and it may be antiferromagnetic or ferromagnetic. The notation for the coupling $-J_{H}$ in $H_{\text {int }}$ is drawn from the Kondo lattice model and has been widely used for the one-orbital FKLM and even in the two-orbital double exchange model [24]. In Kanamori's notation, it should read $-2 J_{H}$, but this notation is mostly used in multiorbital models for TMO. As noticed in previous literature [27,34], a large value of the Hund coupling is appropriate for manganites. We should emphasize however that the model here proposed, as above discussed, is not solely proposed for manganites but for various other compounds and devices. In this sense, the important issue is that the adopted value of $J_{H}$ (see below) leads to the presence of a broad FM region, followed by a phase separated one with dominant AFM correlations, which are the main phases of the 2D FKLM.

To start the study of the effects of the RSOC on the known properties of the FKLM in two dimensions, we set $U=0$ and $J=0$. Actually, it is well known that a large $J_{H}$ prevents double occupancy in one-orbital models so in principle $U=0$ is not very restrictive. In addition, it has been shown that 
FM correlations at intermediate fillings, which are already present for not very large $J_{H}$, are just enhanced by $U[23,25]$. The effect of $U$ at large fillings on phase separation is more involved. It would be expected that a finite $U$ would prevent the tendency to PS but actual calculations have shown that this only occurs for $U \gg J_{H}$ [29], which is a situation outside of our current interest. Interestingly, it was shown that for a spinless two-orbital Hubbard model, valid for the FM phases and large- $J_{H}$ limit of manganites, the kinetic energy of a much simpler single-band model can be used to mimic the one of the $\mathrm{e}_{g}$ model [56]. It is also important to realize that the case $U=0$ is also the relevant one for three-orbital models for $\mathrm{SrTiO}_{3}$ surfaces and interfaces [50-52].

We normalize the hopping and RSOC parameters as $t_{0}^{2}+$ $\lambda_{S O}^{2}=1$ whose square root will be henceforth adopted as the unit of energy. The RSOC implies the movement of electrons and hence it has a kinetic energy associated with it. Then, this normalization keeps the total kinetic energy approximately constant with $\lambda_{S O} / t_{0}$ as is shown in Sec. IV B. The ratio $\lambda_{S O} / t_{0}$ can be considered as the tangent of the angle between the spin-flipping and spin-conserved hoppings. The whole purpose of this normalization is to keep constant the ratio between $J_{H}$ and the total kinetic energy, for a fixed $J_{H}$. Alternatively $t_{0}$ could be kept fixed and adopted as the unit of energy, but in this case a change in $\lambda_{S O}$ would imply an effective change of $J_{H}$ because the total kinetic energy would also change. In this case we would have to deal with the double effect of varying $\lambda_{S O} / t_{0}$ and $J_{H \text {,eff }} / t_{0}$, which would make the analysis less clear. In any case, we have verified that the results do not change qualitatively with both conventions in the range $\lambda_{S O} \leqslant t_{0}$.

We adopted throughout the value of $J_{H}=10$ in this unit, which satisfies the above mentioned requirements, as shown in Sec. IV A. The sole parameters left are then the ratio $\lambda_{s o} / t_{0}$, and the electron filling $v \equiv N_{e} / N(N \equiv L \times L)$.

\section{METHOD}

In this work, we will employ a Monte Carlo technique that is based on the assumption that the localized spins are described by classical continuum spins $\mathbf{S}_{l}=\left(S, \theta_{l}, \phi_{l}\right)$ in spherical coordinates $[25,27,28]$.

The technique works at follows. Starting from a given set of $\theta_{l}, \phi_{l}$, a new configuration is proposed by changing at a given site $j, \theta_{j} \rightarrow \theta_{j}^{\prime}=\theta_{j}+\Delta \theta, \phi_{j} \rightarrow \phi_{j}^{\prime}=\phi_{j}+\Delta \phi$. The new configuration is accepted by computing the difference in total energy $\Delta E=E\left(\theta^{\prime}, \phi^{\prime}\right)-E(\theta, \phi)$ with the usual Metropolis (or Glauber) criterion. For the noninteracting case $(U=0$ in the one-orbital Hamiltonian), the single-particle Hamiltonian is diagonalized using library subroutines and the ground state $\left|\Psi_{0}\right\rangle$ is built by filling the lowest $N_{e}$ states $\left(N_{e}\right.$ is the total number of electrons, see Appendix A). For the interacting case, a full diagonalization of the many-body problem requires the implementation of for example the Lanczos algorithm, which is much costlier than the treatment of the single-particle problem and severely restricts the size of the clusters that can be studied. In both cases, interacting and noninteracting, to diagonalize the Hamiltonian at each site is excessively expensive and we resort to the so-called perturbative Monte
Carlo (PMC) [57-59], in which the full Hamiltonian is only diagonalized after a sweep on the whole lattice.

In this perturbative version, at each sweep a site is chosen (sequentially in the present work) and a change is proposed, $\theta_{j} \rightarrow \theta_{j}^{\prime}$, and $\phi_{j} \rightarrow \phi_{j}^{\prime}$. Now, the difference in energy is computed as $\Delta E=\left\langle\Psi_{0}|\Delta H| \Psi_{0}\right\rangle$, where $\Delta H$ involves only local changes in the Hamiltonian, more specifically in the terms $H_{H}$ and $H_{J}$, which are derived in Appendix B.

For completeness, we would like to emphasize that the present $\mathrm{MC}$ technique is classical. It should not be confused with a quantum $\mathrm{MC}(\mathrm{QMC})$ technique as for example the finitetemperature path-integral auxiliary field algorithm applied in Ref. [60] to the 2D Kondo lattice model. The advantage of this QMC technique is that it could deal with finite Hubbard repulsion $U$ as well as with quantum localized spins. Its main disadvantage is that it is affected by the "minus sign problem", which renders this technique virtually inapplicable to models with fermionic degrees of freedom except when it is performed at half filling as implemented in Ref. [60]. In addition to the above mentioned papers, the classical MC has been widely used for a variety of related models, for example to study the double-exchange model on the pyrochlore lattice [30,31].

Since in this work we limit ourselves to study zerotemperature properties, the Monte Carlo simulation is reduced to a simple optimization procedure, the so-called simulated annealed optimization, in which a Boltzmann weight is used with a parameter that plays the role of temperature. Hence, using the resulting zero-temperature PMC technique, clusters as large as $16 \times 16$ can be studied with conventional desktop computers, although most of the results presented below correspond to the $8 \times 8$ clusters and in some cases we show results for the $12 \times 12$ cluster, just to show that finite-size effects are relatively small. The temperature in the simulated annealing process is lowered from $0.01-0.0008$ in $8-10$ steps, involving a total of $10^{6} \mathrm{MC}$ sweeps with $150000 \mathrm{MC}$ sweeps in the final measurement stage. At least two independent runs were performed for each set of parameters. The energy for the lowest value of the temperature considered differs from the one of the previous value within a relative tolerance of $510^{-5}$ and, in the worst case, it is enough to achieve a relative error of $10^{-4}$ with respect to the exact energy of the FM state starting from a random configuration, close to quarter filling. We have checked the results obtained by the full and the perturbative MC for various electron densities and values of $\lambda_{S O} / t_{0}$, and for various types of boundary conditions.

We have also taken into account different types of boundary conditions (BC). Most of the results reported in the following were obtained for open $\mathrm{BC}$, which avoids problems associated with open and close level shells. To compute transport properties we adopted mixed $\mathrm{BC}$, open in one direction and periodic in the other, which would correspond to a closed strip or ring geometry. Results obtained for fully periodic BC, although noisier, agree reasonably well with the ones obtained with open or mixed BC.

Typical error bars due to PMC statistics are of the size of the symbols employed. Results for the Drude peak strongly depend on the boundary conditions employed and hence the total error for these results are much larger than the PMC errors, as indicated in Sec. IV B. 


\section{RESULTS}

\section{A. Magnetic properties}

In the pure FKLM, various types of magnetic order have been detected in the $J_{H}$-density phase diagram in two dimensions, and this variety is even richer in the presence of an AFM interaction between localized spins $J[27,28]$. As we show in this section, this is also the case when $\lambda_{S O}$ is turned on, even at a fixed $J_{H}=10$ and at zero $J$.

Let us start by examining the total energy as a function of electron density and $\lambda_{S O}$. In Fig. 1, we show the difference between the energy and the energy of the FM state, which is exactly computed by setting $\theta_{l}=0$ in (2), as a function of $v$ and for various values of $\lambda_{S O}$, obtained by PMC for the $8 \times 8$ cluster with open BC. For $\lambda_{S O}=0$, the behavior of the energy is consistent with the known result of a FM order up to $v \lesssim 0.8$. Now, an increasing RSOC makes the energy to increasingly depart from the FM level indicating a departure from the FM order, which can be understood as a lowering of the spinflipping term $H_{0, S O}$ by an AFM localized spin background. This departure in energy is maximal close to quarter filling $(v=0.5)$. For larger fillings, for $\lambda_{S O}=0$ the energy strongly departs from the FM one indicating the proximity to an AFM order that appears due to an effective AFM exchange interaction caused by virtual processes involving $t_{0}$. On the other hand, for a finite $\lambda_{S o}$, the energy starts to get back closer to the one of the FM state in spite of the fact that the peak of the magnetic structure factor (discussed below) continues moving away from $(0,0)$. Notably, close to half filling, the effect of the RSOC is to reduce the tendency to the AFM state. Evidently, the effective exchange due to virtual processes involving $\lambda_{S O}$ would no longer be AFM.

It is interesting also to compare the energies obtained by PMC with the ones obtained for a fixed localized magnetic order that can be plugged in (2) and readily computed.

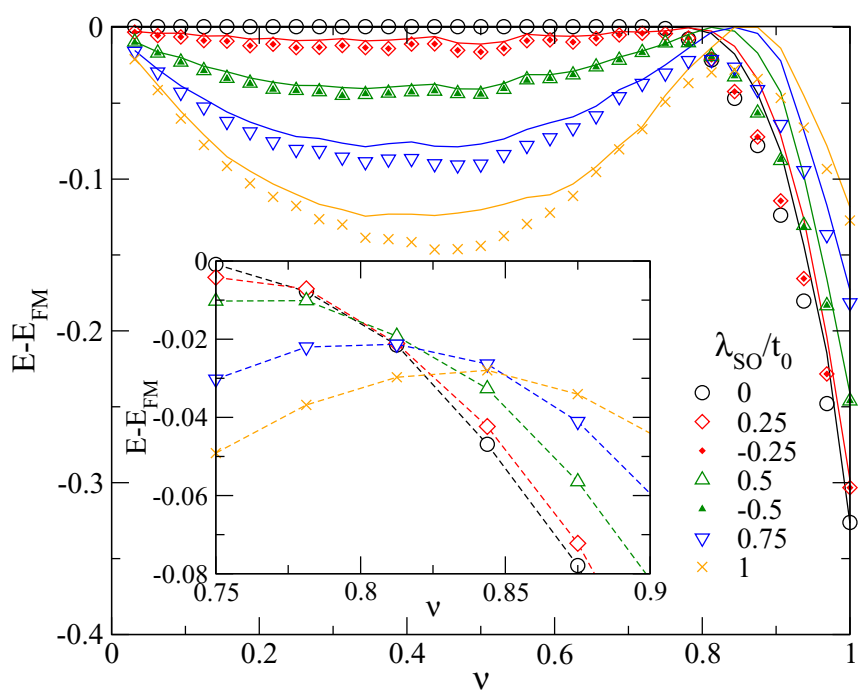

FIG. 1. (Color online) Energy relative to the energy of the FM state, per site, vs electron density for various values of $\lambda_{S o} / t_{0}$. Symbols are PMC results, lines correspond to trial spiral order. The inset is a zoom of the $[0.75,0.9]$ density region for the PMC results only. Results for the $8 \times 8$ cluster with open $\mathrm{BC}$.
In particular, in most of the phase diagram, energies quite close to the PMC ones can be obtained for a generalized spiral order, defined for the classical spin at site $(x, y)$ as $\theta_{x y}=k_{x} x+k_{y} y, \phi_{x y}=\phi_{0}$. In particular we have examined the values $k_{\alpha}=2 n_{\alpha} \pi / L, n_{\alpha}=0, \ldots, L(\alpha=x, y)$, and $\phi_{0}=$ $m \pi / 4, m=0, \ldots, 7$. Diagonal spiral states, $k_{x}=k_{y}$, were mostly previously considered [61], but in the presence of RSOC we found in a large portion of the phase diagram more stable off-diagonal spiral orders as discussed below. Of course the FM (AFM) state correspond to the diagonal spiral state with $k_{x}=k_{y}=0\left(k_{x}=k_{y}=\pi\right)$. For $\lambda_{S O}=0$ all values of $\phi_{0}$ are equivalent, and the energy for a given $\left(k_{x}, k_{y}\right)$ is the same for all the symmetry-equivalent $\mathbf{k}$ points. On the other hand, for $\lambda_{S O}>0$, the optimal energy of the spiral with $\left(k_{x}, k_{y}, \phi_{0}\right)$ is degenerate with the one with $\left(-k_{x},-k_{y}, \phi_{0}+\pi\right)$ but they are different than the optimal energies for the spiral orders with $\left(k_{x},-k_{y}, \phi_{0}+\pi / 2\right)$ and $\left(-k_{x}, k_{y}, \phi_{0}+3 \pi / 2\right)$. This symmetry breaking corresponds to the one observed for the magnetic structure factor as discussed below. The energy of the spiral states have been added to Fig. 1 for comparison. These energies follow the general trend of the ones obtained by PMC. However, the tendency to FM order observed for $v \gtrsim 0.5$ and $\lambda_{S O}>0$ is actually more pronounced for the spiral state, and in fact the FM state is actually recovered for densities in the interval $[0.8,0.9]$ in the range of RSOC values considered.

We have also examined the canted state [22], defined by $\theta_{l}=0$ and $\theta_{0}$ for the two sublattices $\left(\phi_{l}=\phi_{0}\right)$, but we found that its energies are higher than the ones of the generalized spiral state for all the parameter space considered, except when both coincide in the FM or AFM states. This result, for $\lambda_{S O}=0$, is consistent with previous computational studies for FKLM [34].

As it can be seen in Fig. 2(a) the tendency of suppressing the AFM phase, and approaching to the FM state as the density approaches half filling, continues for larger values of $\lambda_{S O} / t_{0}$, including the limiting value $\lambda_{S O} / t_{0}=\infty$, at which the FM is finally reached at $v=1$. Of course this limit cannot be realized in real materials but it is of general mathematical interest. In this plot, results for mixed BC (or strips) on the same $8 \times 8$ cluster are added for comparison for $\lambda_{S O} / t_{0}=1$ and 2 . The irrelevance of the sign of $\lambda_{S O}$ has been checked by a set of independent PMC runs. In Fig. 2(b) results for the $8 \times 8$ and $12 \times 12$ clusters with open $\mathrm{BC}$, and also for the $12 \times 12$ cluster with mixed $\mathrm{BC}$, provide additional evidence that finite-size effects are negligible for these clusters and boundary conditions.

To understand the magnetic behavior suggested by the study of the ground state energies in Figs. 1-2, the spin-spin correlations between localized spins, $C(\mathbf{r})=\left\langle\mathbf{S}_{\mathbf{r}} \cdot \mathbf{S}_{\mathbf{0}}\right\rangle(\mathbf{0}$ is the reference site), and their Fourier transform leading to the static magnetic structure function $\chi(\mathbf{k})$, have been computed.

The RSO coupling leads to a very rich magnetic landscape. To describe the variety of magnetic orders present let us start by examining the results for $\chi(\mathbf{k})$, in the density- $\lambda_{S O} / t_{0}$ plane, depicted in Fig. 3. In Fig. 3(a) we show the modulus of the peak of $\chi(\mathbf{k}),\left|\mathbf{k}_{\text {peak }}\right|$, which essentially describes the proximity to the FM state $\left(\left|\mathbf{k}_{\text {peak }}\right|=0\right)$, or to the AFM state $\left(\left|\mathbf{k}_{\text {peak }}\right|=1\right)$, its maximum value (in units of $\sqrt{2} \pi$ ) for the $8 \times 8$ cluster with open BC. For $\lambda_{S O}=0$ there is a neat crossover from $\mathbf{k}_{\text {peak }}=(0,0)$ to $\mathbf{k}_{\text {peak }}=(\pi, \pi)$ states at $\nu \approx 0.8$, consistently 


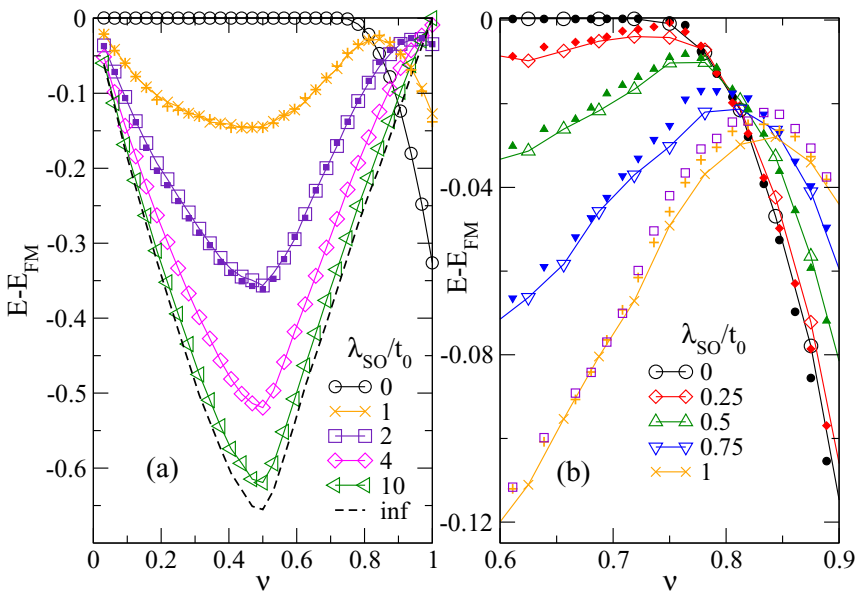

FIG. 2. (Color online) Energy relative to the energy of the FM state vs electron density, per site for various values of $\lambda_{S O} / t_{0}$. (a) $\lambda_{S O} / t_{0} \geqslant 1$ ( $\lambda_{S O}=0$ added for completeness), $8 \times 8$ cluster. Lines and open symbols were obtained for open BC; $\operatorname{stars}\left(\lambda_{s o} / t_{0}=\right.$ 1) and filled squares $\left(\lambda_{S O} / t_{0}=2\right)$ for mixed BC. (b) Comparison of results obtained for the $8 \times 8$ (lines and open symbols) and $12 \times 12$ (filled symbols) clusters with open $\mathrm{BC}$ in the range $0.6<v<0.9$. For $\lambda_{S o} / t_{0}=1$, results for the $12 \times 12$ cluster with mixed $\mathrm{BC}$ (circles) are also included.

with previous studies. However, if for $v \lesssim 0.8, C(\mathbf{r})$ shows an algebraic decay towards a finite value at the maximum distance indicating long range FM order, for $v \gtrsim 0.8, C(\mathbf{r})$ indicates short-range AFM order except at $v=1$ where a long-range AFM order is achieved. In the $v \gtrsim 0.8$ region, the behavior has been explained as a phase-separated AFM-FM state [34] which we discuss below. In the following the term order will refer to at least short-range magnetic order.

As $\lambda_{S O} / t_{0}$ is increased, the peak of the magnetic structure factor departs from both FM and AFM states but remain close to them in the low and high electron density regions respectively, up to $\lambda_{S O} / t_{0} \sim 2$. It is interesting to note that for very large values of the RSOC, $\lambda_{S O} / t_{0} \gtrsim 4$ the situation is reversed, that is, the low- (high-)density region holds now a AFM (FM) state. One should notice however that, as shown in Fig. 3(b),
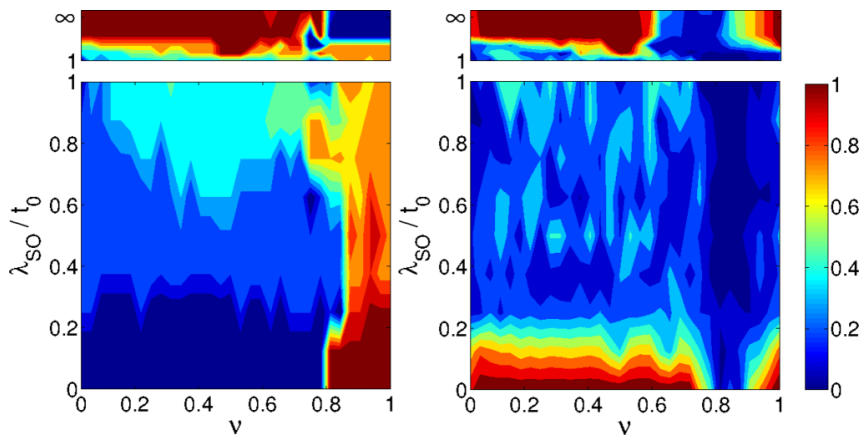

FIG. 3. (Color online) (a) Magnetic phase diagram in the electron density- $\lambda_{S O} / t_{0}$ plane determined from the modulus of the peak of the magnetic structure factor, where dark blue corresponds to FM order $\left(\left|\mathbf{k}_{\text {peak }}\right|=0\right)$ and dark red to AFM order $\left(\left|\mathbf{k}_{\text {peak }}\right|=1\right)$. (b) Intensity of $\chi\left(\mathbf{k}_{\text {peak }}\right)$ in density- $\lambda_{S O} / t_{0}$ plane. Results for the $8 \times 8$ cluster with open BC.
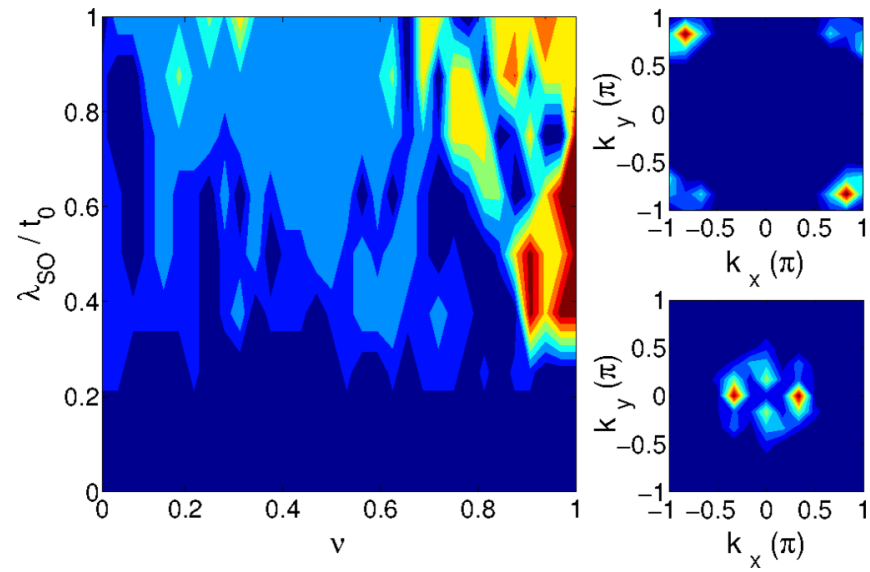

FIG. 4. (Color online) (a) Magnetic phase diagram in the electron density $-\lambda_{S o} / t_{0}$ plane determined from the distance of the peak of $\chi(\mathbf{k})$ to the diagonal line in momentum space (see text), with dark blue (red) corresponding to diagonal (off-diagonal) $\mathbf{k}$, obtained for the $8 \times 8$ cluster with open BC. Right: $\chi(\mathbf{k})$ for $v=0.97$, (top) and $v=0.69$ (bottom), $12 \times 12$ cluster, $\lambda_{S O} / t_{0}=0.5$, with open BC. The color bar code is the same as in Fig. 3

the amplitude of $\chi\left(\mathbf{k}_{\text {peak }}\right)$ is reduced as the RSOC is increased from zero up to $\lambda_{S O} / t_{0}=1$, and then it starts to increase again until recovering its maximum value for $\lambda_{S O} / t_{0}>4$.

At quarter filling, where the physics is dominated by the kinetic energy, as a difference to half filling where it is dominated by the effective AFM exchange interaction, the results of Fig. 3(a) suggest an extension of the effective double-exchange model [21],

$$
H_{0, \mathrm{eff}}=\sum_{l, m}\left(-\tilde{t}_{l m}+\tilde{\lambda}_{l, m}\right) a_{l}^{\dagger} a_{m},
$$

where

$$
\begin{aligned}
\tilde{t}_{l m} & =t_{0} \cos \frac{\theta_{l m}}{2} \\
\tilde{\lambda}_{l, m} & =\lambda_{S O} \sin \frac{\theta_{l m}}{2} .
\end{aligned}
$$

Here $\theta_{l m}$ is the angle between two localized spins at sites $l$ and $m$. Following Ref. [62] it is simple to derive this result at least for the cases when $\theta_{l}-\theta_{m}=0, \pi$ or $\pi / 2$ independently of $\phi_{l}, \phi_{m}$.

It should also be noticed that most of the phase diagram is dominated by magnetic states characterized by an off-diagonal peak of $\chi(\mathbf{k})$, that is, $k_{\text {peak } x} \neq k_{\text {peak }, y}$. This behavior is shown in Fig. 4, where the distance of $\mathbf{k}_{\text {peak }}$ to the diagonal, $d=$ $\left|k_{\text {peak }, x}-k_{\text {peak }, y}\right| / \sqrt{2}$ is plotted in the density- $\lambda_{S O} / t_{0}$ plane. It is apparent that this distance is maximal for intermediate values of $\lambda_{S O} / t_{0}$ and close to half filling. It is interesting also to note that for these cases, the rotational invariance is broken, that is, the PMC simulations are able to select states where the magnetic structure factor is maximal only at two points $\left(k_{x}, k_{y}\right)$, and $\left(-k_{x},-k_{y}\right)$ as shown for two examples, $v=0.97$ (top right panel) and $v=0.69$ (bottom right panel), both for $\lambda_{S O} / t_{0}=0.5$, obtained on the $12 \times 12$ cluster with open BC. This behavior of the magnetic structure factor indicates the presence of a striped magnetic order as it was also previously detected for the FKLM in the presence of a finite exchange 
$J$ between localized spins or for smaller $J_{H}$ [29]. Although the energies are slightly higher, there is in general a good qualitative agreement between the momentum of the optimal spiral state and the momentum of the peak of $\chi(\mathbf{k})$ obtained by PMC at least for densities $v \lesssim 0.75$.

To our knowledge, this is the first report of the influence of the RSOC on the well-known FM-AFM phases in the 2D FKLM.

\section{B. Transport properties}

The optical conductivity is defined as the real part of the linear response to the electric field and can be written as [63]:

$$
\begin{aligned}
\sigma(\omega) & =D \delta(\omega)+\sigma^{\mathrm{reg}}(\omega) \\
& =D \delta(\omega)+\frac{\pi}{L} \sum_{n \neq 0} \frac{\left|\left\langle\Psi_{n}|j| \Psi_{0}\right\rangle\right|^{2}}{E_{n}-E_{0}} \delta\left(\omega-\left(E_{n}-E_{0}\right)\right),
\end{aligned}
$$

where the paramagnetic current along the $x$ direction is:

$$
\begin{aligned}
j & =j_{\mathrm{hop}}+j_{S O} \\
j_{\mathrm{hop}} & =-i e t_{0} \sum_{j, \sigma}\left(c_{j+x \sigma}^{\dagger} c_{j \sigma}-c_{j \sigma}^{\dagger} c_{j+x \sigma}\right) \\
j_{S O} & =i e \lambda_{S O} \sum_{j}\left[\left(c_{j+x \downarrow}^{\dagger} c_{l \uparrow}-c_{l+x \uparrow}^{\dagger} c_{l \downarrow}\right)-\text { H.c. }\right],
\end{aligned}
$$

where $j_{\text {hop }}$ and $j_{S O}$ are the spin-conserving and spin-flipping contributions respectively (the electron charge $e=1$ in the following). The Drude weight $D$ is calculated from the $f$-sum rule as:

$$
\frac{D}{2 \pi}=-\frac{\left\langle H_{0, x}\right\rangle}{2 L}-\frac{1}{L} \sum_{n \neq 0} \frac{\left.\left\langle\Psi_{n}|j| \Psi_{0}\right\rangle\right|^{2}}{E_{n}-E_{0}},
$$

where $K_{x} \equiv-\left\langle H_{0, x}\right\rangle$ is the total kinetic energy of electrons along the $x$ direction.

In order to track the contribution from spin-conserving and spin-flipping transport, from (5), (6), and (7), one can formally define the corresponding quantities for the $\lambda_{S O}=0$ and $t_{0}=0$ limits,

$$
\begin{aligned}
\sigma_{\alpha}(\omega) & =D_{\alpha} \delta(\omega)+\frac{\pi}{L} \sum_{n \neq 0} \frac{\left|\left\langle\Psi_{n}\left|j_{\alpha}\right| \Psi_{0}\right\rangle\right|^{2}}{E_{n}-E_{0}} \delta\left(\omega-\left(E_{n}-E_{0}\right)\right) \\
\frac{D_{\alpha}}{2 \pi} & =-\frac{\left\langle H_{0, \alpha}\right\rangle}{2 L}-\frac{1}{L} \sum_{n \neq 0} \frac{\left.\left\langle\Psi_{n}\left|j_{\alpha}\right| \Psi_{0}\right\rangle\right|^{2}}{E_{n}-E_{0}}
\end{aligned}
$$

with $\alpha=$ hop,SO, respectively. Of course, for nonzero $t_{0}$ and $\lambda_{S O}, \sigma(\omega) \neq \sigma_{\text {hop }}(\omega)+\sigma_{S O}(\omega), D \neq D_{\text {hop }}+D_{S O}$, unless there is no excited state $\left|\Psi_{n}\right\rangle$ such that $\left\langle\Psi_{n}\left|j_{\text {hop }}\right| \Psi_{0}\right\rangle \neq 0$ and $\left\langle\Psi_{n}\left|j_{S o}\right| \Psi_{0}\right\rangle \neq 0$ simultaneously.

Results for the hopping and SO contributions to the kinetic energy per site along the $x$ direction are shown in Figs. 5(a) and 5(c) respectively, and results for the corresponding contributions to the Drude peak, with the above mentioned caveats, in Figs. 5(d) and 5(b). These results present oscillations due to various level crossings in spite of being averaged on the $8 \times 8$ cluster over periodic and strip BC, in this latter case taking phases enclosing magnetic fluxes equal to $0, \pi / 2$ and $\pi$ (twisted BC). In Fig. 5(b) we include the error bars on one point
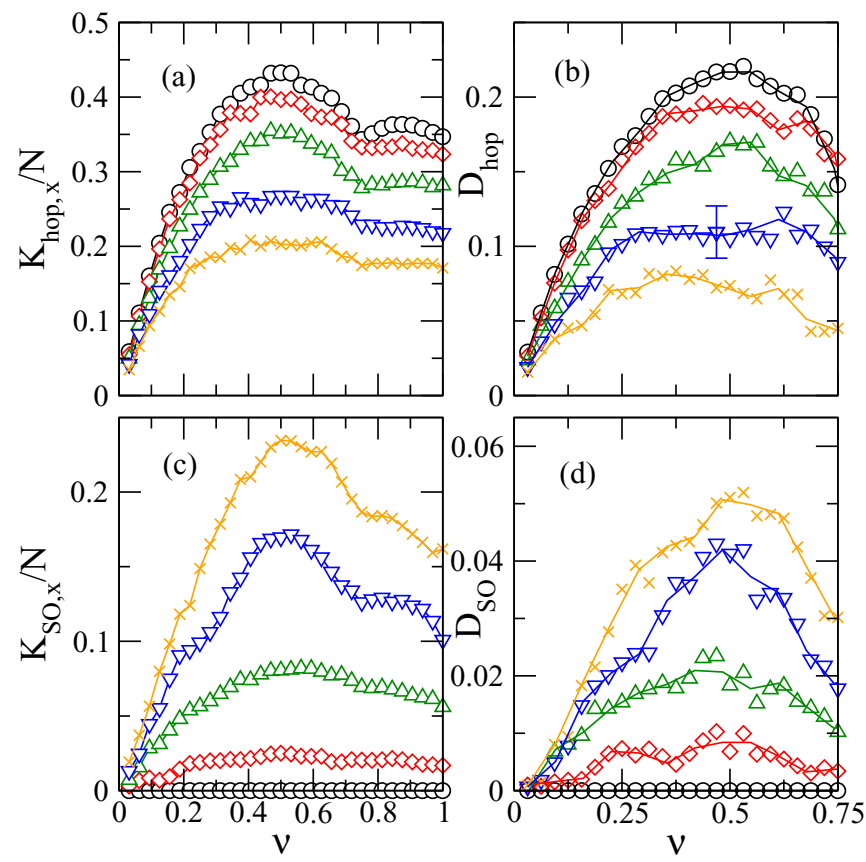

FIG. 5. (Color online) Kinetic energy per site along $x$ direction for (a) the spin-conserving hopping, and (c) the spin flipping or RSOC terms of the Hamiltonian as a function of electron density, for various values of $\lambda_{S O} / t_{0}$. Drude weight for (b) the spin-conserving hopping and (d) the spin flipping or RSOC as a function of electron density, for various values of $\lambda_{S O} / t_{0}$. Symbols and colors are the same as in Fig. 1. Results for the $8 \times 8$ cluster with periodic $\mathrm{BC}$ and strips with twisted BC.

for $\lambda_{S O} / t_{0}=0.75$ is indicative of the dispersion of the values obtained for different BC. Results for the contributions to the Drude peak are only plotted up to densities $v=0.75$, beyond that the total Drude weight, computed according Eq. (7), starts to appreciably deviate from $D_{\text {hop }}+D_{S O}$. Up to this electron filling, $D_{\text {hop }}$ and $D_{S O}$ roughly follows the behavior of $K_{\text {hop }, x}$ and $K_{S O, x}$ respectively.

The total kinetic energy along the $x$ direction, and the total Drude peak, calculated using Eq. (7), are shown in Fig. 6. The total kinetic energy [Fig. 6(a)], for a given density, has very small variation in the range $0 \leqslant \lambda_{S O} / t_{0} \leqslant 1$. On the other hand, the Drude peak is suppressed by increasing RSOC in a monotonous way, within the dispersion of the data previously noticed [Fig. 6(b)]. For $\lambda_{S O} / t_{0}=0$, and for densities $v \lesssim$ 0.75 , the Drude peak is approximately equal to half the kinetic energy per site, $K_{x} / N$, that is, the contribution from the second term in (7) is very small. It is interesting to notice a cusp in $K_{x} / N$ as a function of $v$ at $v \sim 0.75$ suggesting the presence of a crossover in the transport behavior. For densities larger than $v \sim 0.75$, the Drude peak starts to decrease in a rather abrupt fashion, departing from the value of $K_{x} / 2 N$. By observing the density of states (DOS), this crossover suggested by the cusp in $K_{x} / N$ vs $v$, could be related to the Fermi level moving from the bulk of the conduction band towards its high energy edge as $v$ is increased, forming a pseudogap $[28,34]$. Close to $v=1$ this pseudogap separates the conduction band and a small peak at slightly higher energies, which could be considered as an impurity band and hence it gives support to a semiconductor 

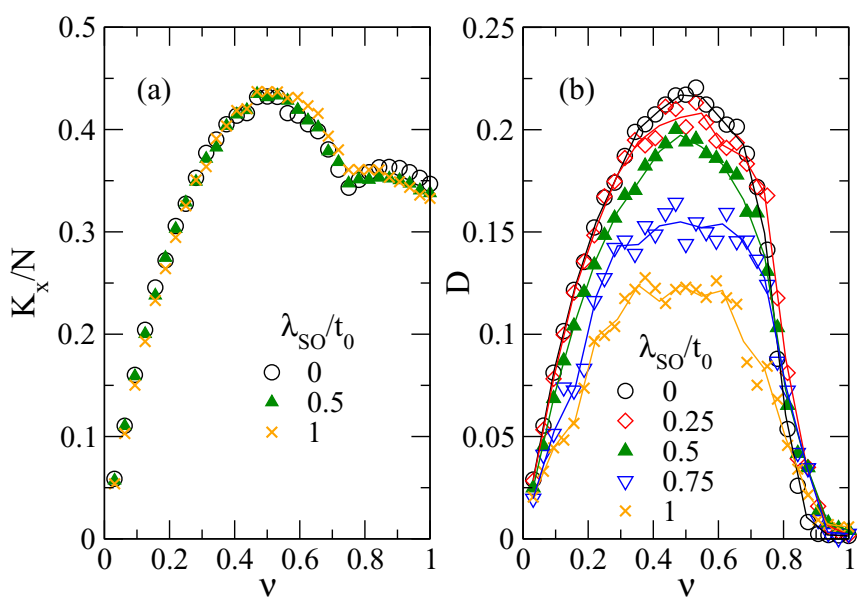

FIG. 6. (Color online) (a) Total kinetic energy along the $x$ direction, and (b) total Drude peak for various values of $\lambda_{S O} / t_{0}$. Lines are guides to the eye. Results for the $8 \times 8$ cluster averaged over periodic $\mathrm{BC}$ and strips with twisted $\mathrm{BC}$.

scenario. These features are well known in the pure FKLM [34] and do not change significantly as the RSOC is turned on.

Another indication of the interplay between magnetic and transport properties can be observed by computing the optical conductivity in the presence of a magnetic field $H$ along the $z$-direction, which is imposed by adding to the Hamiltonian a Zeeman term,

$$
H_{Z}=-H \sum_{l}\left(S_{l}^{z}+s_{l}^{z}\right)
$$

$\mu_{B}=\hbar=1$, and the gyromagnetic factor has been included in $H$. Results obtained for the $8 \times 8$ cluster with strip BC, at quarter filling, and for various values of $\lambda_{S O} / t_{0}$ are shown in Fig. 7. In this figure we show the evolution with $H$ of the hopping and the RSO contributions to the Drude peak, the total Drude weight, and the maximum value of the static magnetic susceptibility. In Fig. 7(a), for $\lambda_{S o} / t_{0}=0.5$, changes in the Drude peaks can be observed around $H \sim 0.02$, where two consecutive crossovers in the peak of $\chi(\mathbf{q})$, first from $(0, \pi / 4)$ to $(0, \pi / 2)$ and then to $(0,0)$ also occur. Similarly for $\lambda_{S O} / t_{0}=1.0$ [Fig. 7(b)], changes in the Drude peak can be observed near $H \sim 0.1$ simultaneously with a change in $k_{\text {peak }}$ from $(0, \pi / 4)$ to $(0,0)$. For $\lambda_{S O} / t_{0}=1.5\left[\right.$ Fig. 7(c) ] $D_{\text {hop }}, D_{S O}$, and $D$ change at $H \sim 0.07$ where $k_{\text {peak }}$ changes from $(\pi, \pi)$ to $(\pi, 0)$, and also at $H \sim 0.25$ where $k_{\text {peak }}$ changes from $(\pi, 0)$ to $(0,0)$. It is clear a general trend at each of those crossovers of increasing (reducing) the hopping (SO) contribution to the Drude peak, although it seems that in most cases the reduction in the $D_{S O}$ is more important than the increase of $D_{\text {hop }}$. This is understandable since the departure of the FM order due to the RSOC is precisely opposed by the magnetic field trying to restore the FM order. We would like to emphasize the fact that the peak of the static magnetic structure factor does not exhaust the richness of the magnetic state. In fact, there are in general many other peaks competing with the one with largest weight. This is the situation for $\lambda_{S O} / t_{0}=1.0$, for $0.1 \lesssim H \lesssim 0.2$ where the pair of peaks $(0, \pi / 4) /(0,-\pi / 4)$ is competing with the dominant one at $(0,0)$.

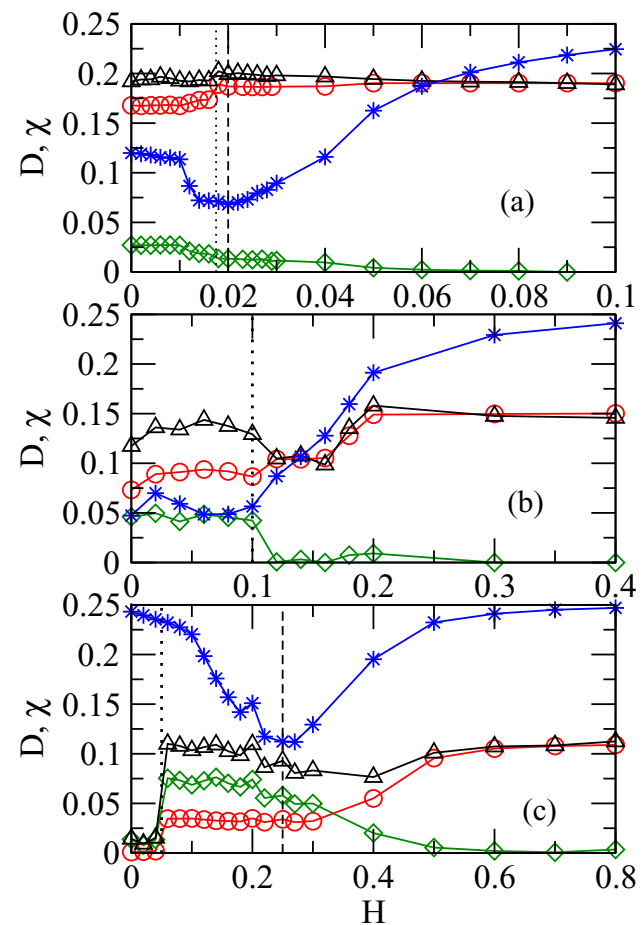

FIG. 7. (Color online) Drude weight $D$ and maximum value of the magnetic structure factor $\chi$, for (a) $\lambda_{S O} / t_{0}=0.5$, (b) 1.0 , and (c) 1.5 at electron density $v=0.5$. The hopping and SO contribution to $D$ are plotted with circles (red) and diamonds (green) respectively. Up triangles (black) and stars (blue) correspond to the total $D$ and $\chi_{\max }$ respectively. $\chi$ has been normalized in this plot in such a way that $\chi_{\max } \leqslant 0.25$. Vertical dotted and dashed lines correspond to changes of the momentum of the peak of $\chi$. Results for the $8 \times 8$ strip BC.

\section{Phase separation}

As emphasized in many previous studies on FKLM [29,34], the behavior of magnetic and transport properties in the high-density region can be understood by the presence of a phase separated state between AFM and FM orders. These two orders correspond to different stable electron fillings, one smaller than $\sim 0.75$ and the other equal to 1 . The electron filling stability is determined by computing the so-called Maxwell construction, which is performed by adding a chemical potential term to the Hamiltonian, $-\mu N_{e}$, where $N_{e}$ is the number of electrons, and determining the electron filling that minimizes the total energy of the resulting Hamiltonian as a function of the chemical potential $\mu$.

Results for the $8 \times 8$ cluster with open $\mathrm{BC}$ are shown in Fig. 8 (left) for various values of $\lambda_{S O} / t_{0}$ in the high electron density region. For $\lambda_{S O}=0$, that is, for the pure FKLM, we recover the well-known phase separated (PS) state [29,34], which extends between densities $v=0.72$ and 1 . As $\lambda_{S O} / t_{0}$ is increased such PS state is gradually suppressed, for example for $\lambda_{S O} / t_{0}=1$, the largest PS state extends between $v=0.82$ and 0.94 . This PS region is further reduced by increasing $\lambda_{S O} / t_{0}$. Qualitatively similar behavior is obtained for the $8 \times 8$ cluster with periodic and mixed BC, and for the $12 \times 12$ cluster with open BC.

This suppression of the PS state can be understood by the fact that the AFM effective exchange, which provides the 


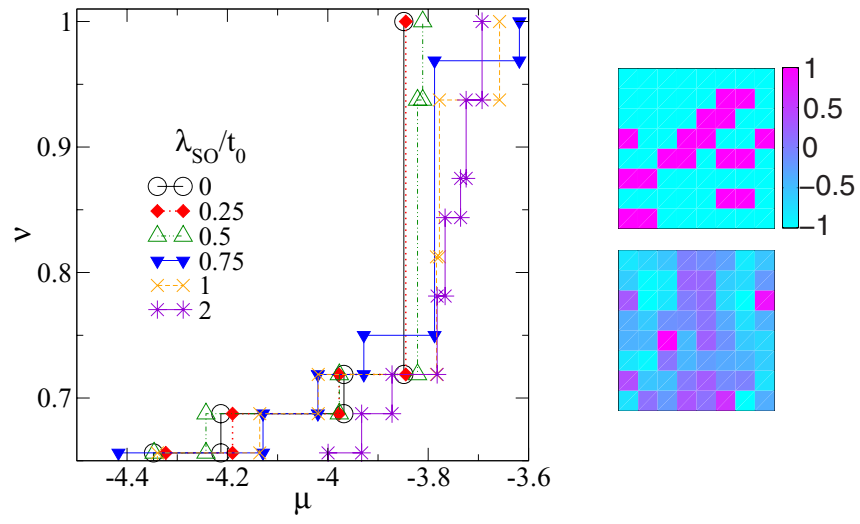

FIG. 8. (Color online) Left: density-chemical potential stability diagram for various values of $\lambda_{S O} / t_{0}$. Right: snapshots of PMC simulations of FM and AFM bonds (see text) for $\lambda_{S O}=0$ (top) and $\lambda_{S o} / t_{0}=1$ (bottom) at $v=0.875$. Results for the $8 \times 8$ cluster with open (left) and periodic (right) BC.

attractive force leading to PS, is suppressed by the RSOC, and even at half filling, the AFM order disappears for $\lambda_{S O} / t_{0} \gtrsim 0.3$ as shown in Fig. 3(a). In fact, the suppression of the PS state is actually related to the substitution of the AFM order by some stripe order with momentum close to $(\pi, \pi)$. This is illustrated in the right panels of Fig. 8 where snapshots of the values of $\mathbf{S}_{l} \cdot \mathbf{S}_{m}$ on each horizontal bond during the PMC simulations, on the $8 \times 8$ with periodic BC are shown. This quantity is normalized in such a way that it is equal to $1(-1)$ for parallel (antiparallel) spins on the bond. Fig. 8 (top panel) shows a neat PS state between FM and AFM regions for $\lambda_{S O}=0$, that is the FKLM, consistently with previous studies. On the other hand, for $\lambda_{S O} / t_{0}=1$, Fig. 8 (bottom panel) shows a very different picture, which illustrates the suppressed PS and the presence of striped states previously discussed. In any case, by relating the FM domains to conducting ones, and the close-to-AFM domains to insulating ones, it becomes understandable that the system behaves for $v \gtrsim 0.75$ as a semiconductor or a poor conductor due to a pseudogap.

\section{Spectral functions}

Finally, we studied the spectral functions corresponding to create or annihilate an electron with momentum $\mathbf{k}, A^{( \pm)}(\mathbf{k}, \omega)$, defined as:

$$
\begin{aligned}
A^{(+)}(\mathbf{k}, \omega) & =\sum_{n, \sigma}\left|\left\langle\Psi_{n}^{(+1)}\left|c_{\mathbf{k} \sigma}^{\dagger}\right| \Psi_{0}\right\rangle\right|^{2} \delta\left(\omega-\left(E_{n}^{(+1)}-E_{0}\right)\right) \\
A^{(-)}(\mathbf{k}, \omega) & =\sum_{n, \sigma}\left|\left\langle\Psi_{n}^{(-1)}\left|c_{\mathbf{k} \sigma}\right| \Psi_{0}\right\rangle\right|^{2} \delta\left(\omega-\left(E_{n}^{(-1)}-E_{0}\right)\right) .
\end{aligned}
$$

$A^{(-)}(\mathbf{k}, \omega)$ probes occupied states, and hence it describes photoemission spectra (PES), while $A^{(+)}(\mathbf{k}, \omega)$ detects unoccupied levels, and it is conventionally ascribed to inverse PES (IPES). The $\delta$ peaks are usually considered after being broadened by a Lorentzian function with width $\epsilon$.

Figure 9 shows the lower or bonding band of the spectra (there is another identical band shifted at higher energies by $\left.J_{H}\right)$ at quarter filling along the line $(0,0) \rightarrow(\pi, 0) \rightarrow$

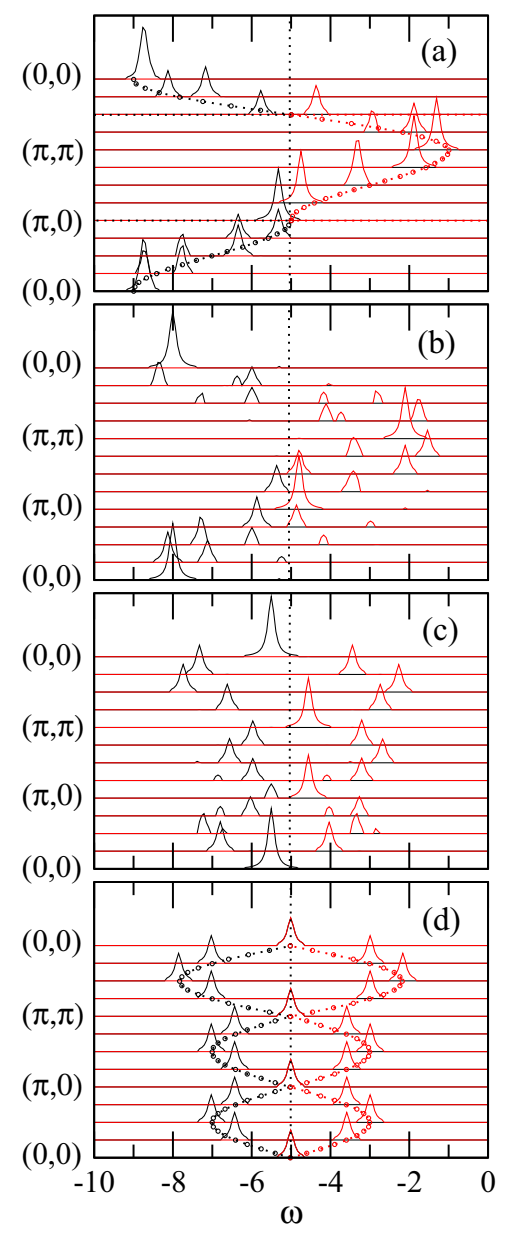

FIG. 9. (Color online) Spectral functions $A^{( \pm)}(\mathbf{k}, \omega)$ for $\lambda_{S O} / t_{0}=$ 0 (a), 0.75 (b), 4.0 (c), and $\infty$ (d), at density $v=0.5$ (bonding band). The PES is plotted with full lines (black) and the IPES with dashed lines (red). The Fermi level is indicated with vertical dotted lines. The Lorentzian width employed is $\epsilon=0.1$. Results obtained for the $8 \times 8$ with periodic BC. Lines with dots in (a) and (d) correspond to the $32 \times 32$ obtained with fixed FM and AFM order of localized spins respectively.

$(\pi, \pi) \rightarrow(0,0)$, obtained for the $8 \times 8$ cluster with periodic BC. For the pure $\operatorname{FKLM}\left(\lambda_{S O}=0\right)$, Fig. 9(a), the shoulder near $(\pi, 0)$ is responsible for the large conductivity at this filling. As $\lambda_{S O} / t_{0}$ increases, the peak at $(0,0)[(\pi, \pi)]$ is shifted to higher (lower) energies thus reducing the number of states at the Fermi level and consequently the conductivity. This reduction of the DOS at the Fermi level could be thought as the opening of a pseudogap, but as it is clear from Fig. 9 there is a restructuring of the Fermi surface as a function of $\lambda_{S O}$. In the limit of $\lambda_{S O} / t_{0}=\infty$, the Fermi surface only touches the Fermi level at the high-symmetry points $(0,0),(\pi, 0),(0, \pi)$, and $(\pi, \pi)$ of the Brillouin zone (BZ).

For a better visualization of the Fermi surface in the limit of $\lambda_{S o} / t_{0}=\infty$, we show in Fig. 10 the energy dispersion $E^{( \pm)}(\mathbf{k})$ obtained from the first peak above the Fermi level for $A^{(+)}(\mathbf{k}, \omega)$ (upper sheet) and the first peak below the Fermi level for $A^{(-)}(\mathbf{k}, \omega)$ (lower sheet). Both PES and IPES sheets touch the Fermi level at the $\Gamma, X, Y$, and $M$ points, the time-reversal invariant momenta of the square BZ [44], consistently with 


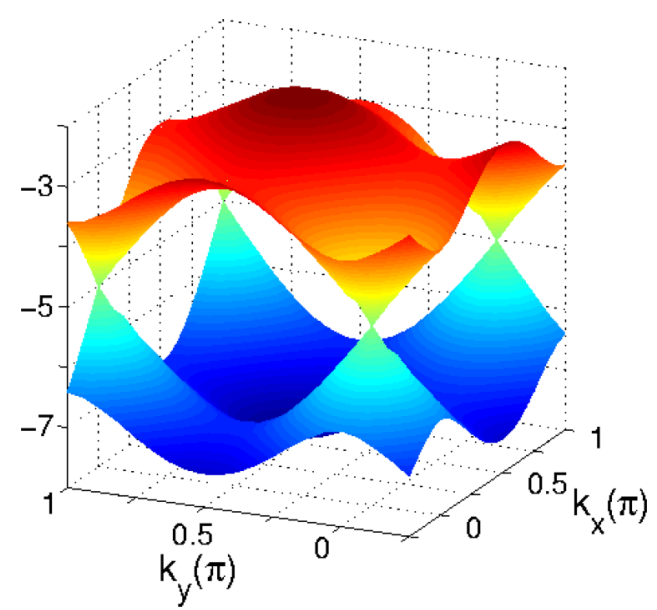

FIG. 10. (Color online) Energy dispersion for $\lambda_{S O}=1, t_{0}=0$, at $v=0.5$, obtained from $A^{( \pm)}(\mathbf{k}, \omega)$. Results for the $32 \times 32$ cluster with AFM fixed localized spin order, periodic BC.

Fig. 9(d). The behavior of $E(\mathbf{k})$ at the $\Gamma, X, Y$, and $M$ points, resembles the features called Dirac cones, since the linear dispersion is equivalent to two-dimensional massless Dirac fermions, which are notably found in graphene [46] but also in other compounds such as pnictides [47] and heavy element compounds [48]. The cones touch the Fermi level, located approximately at $\omega=-5.019$, at their vertices. It is worth to notice that the observed Dirac cones do not appear for the noninteracting $H_{0, S O}$ term in Eq. (2), as it can be readily checked by a simple tight-binding calculation.

The results in Figs. 9 and 10 were obtained for $J=0$, as all results in this work. Within $\mathrm{MC}$ errors, we found the presence of Dirac cones down to $\lambda_{S o} / t_{0}=10$ but so far we have not study this issue systematically. In principle, an AFM $J(J>0)$ should enhance the tendency to an AFM order in the localized spins, and hence one would expect that the Dirac cones could be realized for lower values of $\lambda_{S O} / t_{0}$. Of course, what it really matters is that the conduction electrons have an AFM order, which implies then that also the Hund coupling $J_{H}$ should be large enough. By imposing a fixed AFM order in the localized spins, for $\lambda_{S O}=1, t_{0}=0$, we actually found that Dirac cones with vertices at the Fermi level were present for $J_{H}$ as low as 3 (in the usual units), at quarter filling.

\section{CONCLUSIONS}

In this work we have analyzed the interplay between the Rashba spin-orbit coupling on one side, and the hopping and Hund couplings that characterizes the ferromagnetic Kondo lattice model on the other. Near quarter filling, the RSOC moves the system away from the ferromagnetic metallic state that is present in the pure FKLM, leading to a rich variety of magnetic states and to a loss of conductivity. Near half filling, on the other hand, the mechanism that favors an antiferromagnetic order in the pure FKLM is no longer fully acting in the presence of the RSOC, and the system presents a tendency towards striped magnetic orders. As a consequence the presence of phase separation between antiferromagnetic and ferromagnetic regions in the FKLM is suppressed by the RSOC. In some studies of the effect of strong Rashba coupling on transition metal oxides interfaces, it was reported a tendency towards a phase separated state caused by the RSOC [12]. However in the systems considered the RSOC is proportional to the electron density, a situation that does not correspond to the model studied in the present work. The system still has a very low conductivity in the high-density region in the presence of a RSOC, a characteristic of a semiconductor or a pseudogap, since the DOS is not significantly changed upon switching on the RSOC.

Remarkably, in the limit of RSOC much larger that the hopping integral, the nature of the magnetic states is reversed, that is at quarter filling the system evolves towards an AFM state, and exactly at half filling the system becomes a perfect FM.

Here we report the influence of the Rashba spin-orbit coupling on the well-known ferromagnetic-antiferromagnetic phases in the 2D ferromagnetic Kondo lattice model, particularly important when manganites are involved in interfaces.

The general relationship between conductivity and magnetic order becomes clear at $v=0.5$, particularly when a magnetic field is applied through a Zeeman term. Here, it is clear that a more realistic model for the orbitals involved in the interface as well as more details on the interface are needed to make a comparison with experiments.

The profound effects of the RSOC can also be noticed by examining the spectral functions of creating and annihilating electrons at $v=0.5$. For the pure FKLM, the spectral functions along the main symmetry lines resemble that of a Fermi liquid. On the other hand, in the opposite limit of $\lambda_{S O} \gg t_{0}$ the Fermi surface is reduced to Dirac points located at the $\Gamma, X, Y$, and $M$ points of the Brillouin zone of the square lattice. The observed Dirac cones are a nontrivial feature of the RSOC connected to an AFM background by the Hund coupling.

Future work along this direction includes the search of these features for more realistic sets of parameters better describing the complexity of TMOs surfaces and interfaces. The rich physics of these interface systems, including superconductivity [10] and spin-Hall effect will also be addressed in future work.

\section{ACKNOWLEDGMENTS}

We would like to thank Elbio Dagotto for valuable comments and suggestions. The authors are partially supported by the Consejo Nacional de Investigaciones Científicas y Técnicas (CONICET) of Argentina.

\section{APPENDIX A: HILBERT SPACE}

The Rashba spin-orbit term, and actually also the Hund's term after the classical localized spins assumption leading to (B1), makes the $z$ projection of the total spin, $S_{\text {total }}^{z}$, no longer a good quantum number.

In the noninteracting case, in real space, the single-particle Hamiltonian has to be formulated in the space of spin up and down electrons, thus becoming a $2 N \times 2 N$ matrix ( $N$ is the number of cluster sites).

In the interacting case, the Hilbert space has to include all possible values of $S_{\text {total }}^{z}$, from $-N_{e} / 2$ to $N_{e} / 2$, thus increasing the difficulty in reaching large cluster sizes. For example, in the $4 \times 4$ cluster, at quarter filling, the dimension of the Hilbert space is 10518300 . 


\section{APPENDIX B: VARIATION OF THE HAMILTONIAN}

It is easy to prove that the Hund coupling between localized and conduction electron spins can be written as:

$$
\begin{aligned}
\mathbf{S}_{l} \cdot \mathbf{s}_{l \alpha}= & \frac{S}{2} \cos \theta_{l}\left(n_{l \alpha \uparrow}-n_{l \alpha \downarrow}\right) \\
& +S \sin \theta_{l}\left(e^{i \phi_{l}} c_{l \alpha \downarrow}^{\dagger} c_{l \alpha \uparrow}+\text { H.c. }\right) .
\end{aligned}
$$

In the same way, the AFM coupling between localized spins can be written as:

$$
\mathbf{S}_{l} \cdot \mathbf{S}_{m}=S^{2}\left[\sin \theta_{l} \sin \theta_{m} \cos \left(\phi_{l}-\phi_{m}\right)+\cos \theta_{l} \cos \theta_{m}\right]
$$

Then, the variation of the $H_{H}$ term results:

$$
\begin{aligned}
\Delta H_{H}(l)= & J_{H} S \sum_{\alpha} \frac{1}{2}\left(\cos \theta_{l}^{\prime}-\cos \theta_{l}\right)\left(n_{l \alpha \uparrow}-n_{l \alpha \downarrow}\right) \\
& \left.+\left[\left(\sin \theta_{l}^{\prime} e^{i \phi_{l}^{\prime}}-\sin \theta_{l} e^{i \phi_{l}}\right) c_{l \alpha \downarrow}^{\dagger} c_{l \alpha \uparrow}+\text { H.c. }\right)\right] .
\end{aligned}
$$

The variation of the $H_{J}$ term is:

$$
\begin{aligned}
\Delta H_{J}(l)= & J S^{2} \sum_{m(l)} \sin \theta_{m}\left[\sin \theta_{l}^{\prime} \cos \left(\phi_{m}-\phi_{l}^{\prime}\right)\right. \\
& \left.-\sin \theta_{l} \cos \left(\phi_{m}-\phi_{l}\right)\right] \\
& +\cos \theta_{m}\left(\cos \theta_{l}^{\prime}-\cos \theta_{m}\right) .
\end{aligned}
$$

[1] H. Y. Hwang, Y. Iwasa, M. Kawasaki, B. Keimer, N. Nagaosa, and Y. Tokura, Nature Mater. 11, 103 (2012).

[2] E. I. Rashba, Sov. Phys. Solid State 2, 1109 (1960); Y. A. Bychkov and E. I. Rashba, J. Phys. C 17, 6039 (1984).

[3] M. I. Dyakonov and V. I. Perel, Phys. Lett. A 35, 459 (1971).

[4] J. E. Hirsch, Phys. Rev. Lett. 83, 1834 (1999).

[5] R. Winkler, Spin-orbit Coupling Effects in Two-Dimensional Electron and Hole Systems (Springer, Berlin, 2003).

[6] S. A. Wolf, D. D. Awschalom, R. A. Buhrman, J. M. Daughton, S. von Molnar, M. L. Roukes, A. Y. Chtchelkanova, and D. M. Treger, Science 294, 1488 (2001).

[7] G. A. Prinz, Science 282, 1660 (1998).

[8] D. Awschalom, Physics 2, 50 (2009).

[9] A. D. Caviglia, M. Gabay, S. Gariglio, N. Reyren, C. Cancellieri, and J.-M. Triscone, Phys. Rev. Lett. 104, 126803 (2010).

[10] M. Ben Shalom, M. Sachs, D. Rakhmilevitch, A. Palevski, and Y. Dagan, Phys. Rev. Lett. 104, 126802 (2010).

[11] Y. Kim, R. M. Lutchyn, and C. Nayak, Phys. Rev. B 87, 245121 (2013).

[12] S. Caprara, F. Peronaci, and M. Grilli, Phys. Rev. Lett. 109, 196401 (2012).

[13] S. Banerjee, O. Erten, and M. Randeria, Nat. Phys. 9, 626 (2013).

[14] K. Gopinadhan, A. Annadi, Y. Kim, A. Srivastava, Ariando, and T. Venkatesan, arXiv:1311.7532.

[15] C. Şahin, G. Vignale, and M. E. Flatté, Phys. Rev. B 89, 155402 (2014).

[16] I. M. Miron, G. Gaudin, S. Auffret, B. Rodmacq, A. Schuhl, S. Pizzini, J. Vogel, and P. Gambardella, Nature Mater. 9, 230 (2010).

[17] X. Wang and A. Manchon, Phys. Rev. Lett. 108, 117201 (2012).

[18] D. A. Pesin and A. H. Mac Donald, Phys. Rev. B 86, 014416 (2012).

[19] H. Nakamura, T. Koga, and T. Kimura, Phys. Rev. Lett. 108, 206601 (2012).

[20] P. D. C. King, R. H. He, T. Eknapakul, P. Buaphet, S.-K. Mo, Y. Kaneko, S. Harashima, Y. Hikita, M. S. Bahramy, C. Bell, Z. Hussain, Y. Tokura, Z.-X. Shen, H. Y. Hwang, F. Baumberger, and W. Meevasana, Phys. Rev. Lett. 108, 117602 (2012).
[21] C. Zener, Phys. Rev. 81, 440 (1951); P. W. Anderson and H. Hasegawa, ibid. 100, 675 (1955).

[22] P. G. de Gennes, Phys. Rev. 118, 141 (1960).

[23] J. Riera, K. Hallberg, and E. Dagotto, Phys. Rev. Lett. 79, 713 (1997).

[24] J. van den Brink and D. Khomskii, Phys. Rev. Lett. 82, 1016 (1999).

[25] E. Dagotto, S. Yunoki, A. L. Malvezzi, A. Moreo, J. Hu, S. Capponi, D. Poilblanc, and N. Furukawa, Phys. Rev. B 58, 6414 (1998).

[26] Q. Si, J. H. Pixley, E. Nica, S. J. Yamamoto, P. Goswami, R. Yu, and S. Kirchner, arXiv:1312.0764 [J. Phys. Soc. Jpn. (to be published)].

[27] S. Yunoki, J. Hu, A. L. Malvezzi, A. Moreo, N. Furukawa, and E. Dagotto, Phys. Rev. Lett. 80, 845 (1998).

[28] A. Moreo, S. Yunoki, and E. Dagotto, Phys. Rev. Lett. 83, 2773 (1999).

[29] E. Dagotto, T. Hotta, and A. Moreo, Phys. Rep. 344, 153 (2001).

[30] Y. Motome and N. Furukawa, Phys. Rev. B 82, 060407 (2010).

[31] H. Ishizuka and Y. Motome, Phys. Rev. B 88, 100402(R) (2013).

[32] A. Chattopadhyay, A. J. Millis, and S. Das Sarma, Phys. Rev. B 64, 012416 (2001).

[33] C. Santos and W. Nolting, Phys. Rev. B 65, 144419 (2002).

[34] E. Dagotto, Nanoscale Phase Separation in Manganites (Springer-Verlag, Heidelberg, 2002).

[35] At least in one dimension this assumption was checked against density matrix-renormalization group calculations using $S=$ 3/2 localized spins in Ref. [25].

[36] M. Daghofer, A. M. Oleś, D. R. Neuber, and W. von der Linden, Phys. Rev. B 73, 104451 (2006).

[37] Lu Li, C. Richter, S. Paetel, T. Kopp, J. Mannhart, and R. C. Ashoori, Science 332, 825 (2011).

[38] X.-Y. Feng, J. Dai, C.-H. Chung, and Q. Si, Phys. Rev. Lett. 111, 016402 (2013).

[39] C. D. Hu, Phys. Rev. B 75, 172106 (2007).

[40] H. Watanabe, T. Shirakawa, and S. Yunoki, Phys. Rev. Lett. 110 , 027002 (2013).

[41] M. Behrmann, C. Piefke, and F. Lechermann, Phys. Rev. B 86, 045130 (2012).

[42] J. A. Riera, Phys. Rev. B 88, 045102 (2013).

[43] M. Z. Hasan and C. L. Kane, Rev. Mod. Phys. 82, 3045 (2010). 
[44] Y. Ando, J. Phys. Soc. Jpn. 82, 102001 (2013).

[45] H. Zhang, C. X. Liu, X.-L. Qi, X. Dai, Z. Fang, and S. C. Zhang, Nat. Phys. 5, 438 (2009).

[46] A. H. Castro Neto, F. Guinea, N. M. R. Peres, K. S. Novoselov, and A. K. Geim, Rev. Mod. Phys. 81, 109 (2009).

[47] K. K. Huynh, Y. Tanabe, and K. Tanigaki, Phys. Rev. Lett. 106, 217004 (2011).

[48] M. N. Ali, Q. D. Gibson, T. Klimczuk, and R. J. Cava, Phys. Rev. B 89, 020505(R) (2014).

[49] I. V. Solovyev, Phys. Rev. B 83, 054404 (2011).

[50] G. Khalsa, B. Lee, and A. H. MacDonald, Phys. Rev. B 88, 041302 (2013).

[51] D. Bucheli, M. Grilli, F. Peronaci, G. Seibold, and S. Caprara, Phys. Rev. B 89, 195448 (2014).

[52] J. Ruhman, A. Joshua, S. Ilani, and E. Altman, arXiv:1311.4541.

[53] K. V. Shanavas and S. Satpathy, Phys. Rev. Lett. 112, 086802 (2014).
[54] J. D. Sau, R. M. Lutchyn, S. Tewari, and S. Das Sarma, Phys. Rev. Lett. 104, 040502 (2010).

[55] T. P. Pareek and P. Bruno, Phys. Rev. B 65, 241305 (2002).

[56] L. F. Feiner and A. M. Oleś, Phys. Rev. B 71, 144422 (2005).

[57] S. Duane, A. D. Kennedy, B. J. Pendleton, and D. Roweth, Phys. Lett. B 195, 216 (1987).

[58] T. N. Troung and E. V. Stefanovich, Chem. Phys. Lett. 256, 348 (1996).

[59] M. P. Kennett, M. Berciu, and R. N. Bhatt, Phys. Rev. B 66, 045207 (2002).

[60] S. Capponi and F. F. Assaad, Phys. Rev. B 63, 155114 (2001).

[61] J. Inoue and S. Maekawa, Phys. Rev. Lett. 74, 3407 (1995).

[62] Y. A. Izyumov and Y. N. Skryabin, Phys.-Usp. 44, 109 (2001).

[63] R. M. Fye, M. J. Martins, D. J. Scalapino, J. Wagner, and W. Hanke, Phys. Rev. B 44, 6909 (1991). 